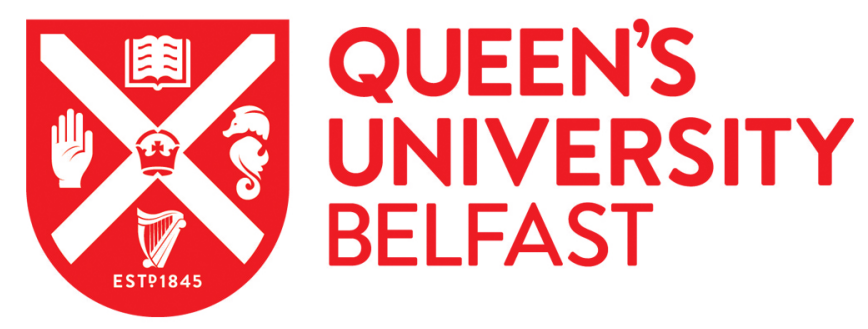

\title{
Saturated fat intake and prostate cancer aggressiveness:results from the population-based North Carolina-Louisiana Prostate Cancer Project
}

Allott, E. H., Arab, L., Su, L. J., Farnan, L., Fontham, E. T. H., Mohler, J. L., Bensen, J. T., \& Steck, S. E. (2017). Saturated fat intake and prostate cancer aggressiveness:results from the population-based North CarolinaLouisiana Prostate Cancer Project. Prostate Cancer and Prostatic Diseases, 20(1), 48-54.

https://doi.org/10.1038/pcan.2016.39

Published in:

Prostate Cancer and Prostatic Diseases

Document Version:

Peer reviewed version

Queen's University Belfast - Research Portal:

Link to publication record in Queen's University Belfast Research Portal

Publisher rights

(C) 2018 Springer Nature Limited. All rights reserved.

Saturated fat intake and prostate cancer aggressiveness: results from the population-based North Carolina-Louisiana Prostate Cancer Project

\section{General rights}

Copyright for the publications made accessible via the Queen's University Belfast Research Portal is retained by the author(s) and / or other copyright owners and it is a condition of accessing these publications that users recognise and abide by the legal requirements associated with these rights.

Take down policy

The Research Portal is Queen's institutional repository that provides access to Queen's research output. Every effort has been made to ensure that content in the Research Portal does not infringe any person's rights, or applicable UK laws. If you discover content in the

Research Portal that you believe breaches copyright or violates any law, please contact openaccess@qub.ac.uk. 
Saturated fat intake and prostate cancer aggressiveness: results from the population-based North Carolina-Louisiana Prostate Cancer Project

Emma H. Allott, Ph.D. ${ }^{1,2}$, Lenore Arab, Ph.D. ${ }^{3}$, L. Joseph Su, Ph.D. ${ }^{4}$, Laura Farnan, Ph.D. ${ }^{2}$, Elizabeth T.H. Fontham, Ph.D. ${ }^{5}$, James L. Mohler, M.D. ${ }^{2,6,7}$, Jeannette T. Bensen, Ph.D. ${ }^{1,2}$ Susan E. Steck, Ph.D. ${ }^{8}$

${ }^{1}$ Department of Nutrition, University of North Carolina at Chapel Hill, Chapel Hill, NC

${ }^{2}$ Lineberger Comprehensive Cancer Center, University of North Carolina at Chapel Hill, Chapel Hill, NC

${ }^{3}$ David Geffen School of Medicine, University of California Los Angeles, Los Angeles, CA

${ }^{4}$ Winthrop P Rockefeller Cancer Institute and College of Public Health, University of Arkansas for Medical Sciences, Little Rock, AR

${ }^{5}$ School of Public Health, Louisiana State University Health Sciences Center, New Orleans, LA ${ }^{6}$ Department of Urology, Roswell Park Cancer Institute, Buffalo, NY

${ }^{7}$ Department of Urology, University of North Carolina at Chapel Hill, Chapel Hill, NC

${ }^{8}$ Department of Epidemiology and Biostatistics, University of South Carolina, Columbia, SC

Running title: Saturated fat and aggressive prostate cancer

Corresponding author: Dr. Emma H. Allott, Department of Nutrition, CB 7461, University of North Carolina at Chapel Hill, 135 Dauer Drive, Chapel Hill, NC 27599, USA. Phone: +1 919966-7230; Fax: +1 919-966-2089; E-mail: allott@email.unc.edu 
Financial support: This work was supported by the American Institute for Cancer Research (E.

H. Allott). The North Carolina-Louisiana Prostate Cancer Project (PCaP) was funded by the Department of Defense contract DAMD 17-03-2-0052.

Number of words: 2,875 (text) +299 (abstract) $=3,174$; Number of Tables: 4; Number of Figures: 0 


\begin{abstract}
Background: Epidemiologic and laboratory evidence supports a role for cholesterol in prostate cancer (PC). Dietary saturated fat content impacts serum cholesterol levels. However, epidemiologic associations between saturated fat and PC aggressiveness are inconsistent. We hypothesized that high saturated fat intake would be associated with increased PC aggressiveness, and that statin use would modify this association.
\end{abstract}

Methods: Of 1,854 PC cases in the North Carolina-Louisiana PC Project (PCaP), 321 (17\%) were classified as high aggressive [Gleason sum $\geq 8$, PSA $>20 \mathrm{ng} / \mathrm{ml}$, or (Gleason sum $\geq 7$ and clinical stage T3-4)] or low/intermediate aggressive (all other cases). Using low/intermediate aggressive cases as the referent group, we examined the association between tertiles of total-fat adjusted saturated fat intake and high aggressive PC using logistic regression, overall and stratified by race and statin use. We examined total fat-adjusted polyunsaturated and monounsaturated fatty acids (PUFA and MUFA, respectively), trans fat, and cholesterol in secondary analysis.

Results: High total fat-adjusted saturated fat intake was associated with an elevated odds ratio (OR) for aggressive PC (ORT3vs.T1 1.51; 95\% CI 1.10-2.06; $\mathrm{p}$-trend=0.009), with an attenuated association in statin users (ORT3vs.T1 1.16 ; 95\% CI 0.67-2.01; $\mathrm{p}$-trend=0.661) compared to nonusers $\left(\mathrm{OR}_{\mathrm{T} 3 \mathrm{vs.T1}} 1.71 ; 95 \%\right.$ CI 1.16-2.51; $\mathrm{p}$-trend=0.053). High total fat-adjusted cholesterol intake was associated with aggressive PC in European Americans (ORT3vs.T1 1.62; 95\% CI 1.022.58; $\mathrm{p}$-trend=0.056), but not African Americans (ORT3vs.T1 0.92; 95\% CI 0.60-1.42; ptrend=0.750). High total fat-adjusted PUFA was inversely associated with PC aggressiveness (ORT3vs.T1 0.75; 95\% CI 0.55-1.03), although this was not significant. No associations were found between total fat-adjusted MUFA or trans fat and PC aggressiveness. 
Conclusions: High total fat-adjusted saturated fat intake was associated with increased PC aggressiveness, with a suggestion of a stronger effect in men not using statins. The association between total fat-adjusted cholesterol intake and PC aggressiveness was most pronounced in European Americans. 


\section{Introduction}

Prostate cancer (PC) incidence rates vary more than 25 -fold worldwide, and are highest in Western countries ${ }^{1}$. International variation in incidence rates is due in part to differences in screening practices between countries, but dietary factors may also play a role. Dietary fat intake, unlike other macronutrients, varies more than fivefold worldwide, and Western populations are among the highest consumers of saturated and trans fats, and cholesterol ${ }^{2}$.

High fat dietary interventions in mouse models of PC drive tumor growth ${ }^{3,4}$, but several large prospective cohort studies found no association between different types of dietary fat and PC risk $^{5-7}$. However, some evidence supports an association between elevated intake of saturated fat and increased PC aggressiveness ${ }^{8,9}$ and mortality ${ }^{8,10,11}$. Many prior studies were limited by incomplete assessment of potential confounders, including PC screening ${ }^{7}$. In addition, prior studies were primarily carried out in white populations, and so the impact of race on these associations remains poorly understood.

Using the North Carolina-Louisiana PC Project (PCaP), a case-only study of incident PC in European Americans (EAs) and African Americans (AAs), we examined associations between dietary saturated fat intake and PC aggressiveness, overall and stratified by race. Dietary saturated fat intake impacts serum cholesterol levels ${ }^{12}$, and epidemiologic evidence supports a role for serum cholesterol and cholesterol-lowering statins in $\mathrm{PC}^{13,14}$. Indeed, we previously reported an inverse association between statin use and $\mathrm{PC}$ aggressiveness in $\mathrm{PCaP}^{15}$. As such, we explored the hypothesis that the association between saturated fat and PC aggressiveness would be weaker in men using statins to control serum cholesterol levels. In secondary analysis, we examined associations between total fat, polyunsaturated fatty acid (PUFA), monounsaturated 
fatty acid (MUFA), trans fat, cholesterol intake and PC aggressiveness. 


\section{Materials and Methods}

\section{Study population}

PCaP enrolled men 40-79 years of age diagnosed with PC on or after July 1, 2004, as previously described ${ }^{16}$. Research subjects self-identified as either AA/black or Caucasian/white (EA) in response to the open-ended question "What is your race?" Recruitment ended October 2007 in North Carolina (NC) and July 2009 in Louisiana (LA). Research protocols were approved by the institutional review boards at the University of North Carolina, Louisiana State University Health Services Center, and Department of Defense PC Research Program.

\section{Exposure assessment and explanatory variables}

PCaP research nurses administered questionnaires during an in-home visit approximately three months after diagnosis ${ }^{16}$. PC screening frequency was classified as a three-level variable (never screened, $\leq 1$ PSA and/or digital rectal exam (DRE) per year, $>1$ PSA and/or DRE per year), as previously described ${ }^{15}$. The dietary assessment instrument was a modified National Cancer Institute (NCI) Diet History Questionnaire (DHQ) ${ }^{17,18}$ to which Southern US foods were added. Research subjects reported dietary intake during the 12 month period prior to PC diagnosis. Dietary intake of fatty acids and cholesterol was calculated using Diet*Calc analysis software (version 1.4.3, NCI, Applied Research Program, November 2005). No gold standard exists for validating calories from dietary fats and cholesterol. However, a comparative validation study indicated that energy-adjusted correlations between multiple 24 hour recalls and DHQ estimates were 0.68 for saturated fat, 0.61 for PUFA, 0.60 for MUFA, 0.66 for total fat, and 0.64 for cholesterol ${ }^{17}$. Research subjects gathered all prescription medications used in the 2-week period prior to interview and presented them to the research nurse at the time of interview for 
documentation of current statin use. We previously reported an inverse association between statin use and PC aggressiveness, with no differences in this association by statin type or dose ${ }^{15}$. Therefore, we did not consider statin type or dose in the present analysis.

\section{Outcome assessment}

Clinical stage, biopsy Gleason sum, and serum PSA at diagnosis were abstracted from medical records. High aggressive PC, our outcome of interest, was defined as Gleason sum $\geq 8$, or PSA $>20 \mathrm{ng} / \mathrm{ml}$, or (Gleason sum $\geq 7$ and clinical stage T3-T4). Low/intermediate aggressive PC (all other cases) was used as the referent group for all analyses ${ }^{16}$. We excluded 85 research subjects lacking sufficient clinical data to define a PC aggressiveness category. We also excluded research subjects missing body mass index (BMI; $n=21)$, PC screening frequency $(n=221)$, smoking status $(\mathrm{n}=1)$, and those who reported implausibly low or high caloric intake $(<800$ or $\geq 6,000 \mathrm{kcal} /$ day, respectively; $\mathrm{n}=76$ ). These exclusions resulted in 1,854 research subjects ( $\mathrm{n}=993$ EA and $\mathrm{n}=861$ AA) for the present analysis.

\section{Statistical analysis}

We examined differences in patient and tumor characteristics between low/intermediate versus high aggressive PC cases, and across tertiles of total fat-adjusted saturated fat intake, using chisquare tests for categorical variables, student's t-tests for continuous, normally-distributed variables and rank sum tests for continuous non-normally distributed variables.

Dietary variables were log transformed to improve normality. Saturated fat intake was adjusted for total fat intake using the residual method to examine the impact of saturated fat on PC aggressiveness independent of total fat intake ${ }^{19}$. Total fat-adjusted saturated fat intake was analyzed as a categorical variable, based on tertiles of the distribution among low/intermediate 
aggressive PC cases. Logistic regression was used to estimate multivariable-adjusted odds ratios (ORs) and 95\% confidence intervals (CIs) for associations between tertiles of total fat-adjusted saturated fat intake and PC aggressiveness (high vs. low/intermediate) using the lowest tertile (T1) as the referent. In secondary analysis, we explored race-specific tertiles of total fat-adjusted saturated fat intake. For multivariable analysis, we selected covariates using a directed acyclic graph and performed backwards selection to build our final model which included age (continuous), race (AA, EA), site (NC, LA), marital status (married/living as married, divorced/separated/widowed, single), BMI (continuous), statin use (yes, no), smoking status (never, former, current), PC screening frequency (never, $\leq 1$ screening test per year, $>1$ screening test per year), energy intake (kcal; continuous), and energy-adjusted total fat intake (calculated using the residual method). Physical activity, education, income, Charleson comorbidity index, and family history of PC were dropped by backwards selection and therefore not included in the final model. Tests for trend of categorical variables were conducted by assigning each research subject the median value of their tertile of intake and modeling these values as a continuous variable. In secondary analysis, using the same method as described for total fat-adjusted saturated fat, we examined associations for tertiles of total fat-adjusted PUFA, MUFA, trans fat, and cholesterol intake and for tertiles of energy-adjusted total fat intake. We tested for interaction between race and dietary fat intake for predicting PC aggressiveness by incorporating a cross product term into the logistic regression model, and calculating the global p-value of the interaction term using the Wald test. We explored statin use as an effect modifier of associations between total fat-adjusted saturated fat intake and PC aggressiveness using models stratified by statin use. 
Statistical analyses were performed using Stata 13.1 (Stata, Corp., College Station, TX, USA). Statistical significance was two-sided with $p<0.05$. 


\section{Results}

Demographic characteristics by PC aggressiveness

Research subjects with high aggressive PC tended to be older at diagnosis, and were more likely to be AA than EA (Table 1). High aggressive PC cases were less likely to be married/living as married, less likely to report a history of PC screening, and had lower education and income levels than low/intermediate cases. Alcohol intake and level of physical activity did not differ by PC aggressiveness (data not shown), but research subjects with high aggressive disease were more likely to be current smokers and obese, and less likely to be statin users.

Saturated fat made up a larger percentage of total fat intake and PUFA made up a smaller percentage of total fat intake in research subjects with high versus low/intermediate aggressive PC, but there were no differences in percent MUFA or trans fat intake by PC aggressiveness (Table 1). Relative to research subjects with low/intermediate aggressive PC, those with high aggressive PC consumed a greater percentage of calories from total fat, and had higher cholesterol intake and higher daily energy intake. Dietary fat, cholesterol and energy intake by race are presented in Supplementary Table 1.

\section{Demographic and tumor characteristics by tertiles of total fat-adjusted saturated fat intake}

Age at diagnosis did not differ by saturated fat intake, but AAs consumed less saturated fat than EAs (Table 2). Research subjects who consumed greater amounts of saturated fat were more likely to have high aggressive PC, less likely to be married/living as married and less likely to report a history of PC screening. Level of education, income, smoking, and obesity status did not differ across tertiles of total fat-adjusted saturated fat intake. 
Total fat-adjusted saturated fat, PUFA, MUFA and trans fat intake and PC aggressiveness

Among 1,854 PC cases, 321 ( $\mathrm{n}=175$ AA and n=146 EA) had high aggressive disease. Relative to the lowest tertile, research subjects in the upper tertile of total fat-adjusted saturated fat intake had an elevated OR for high aggressive PC following adjustment for potential confounders $\left(\mathrm{OR}_{\mathrm{T} 3}\right.$ vs. T1 1.51; 95\% CI 1.10-2.06; $p$-trend $=0.009$; Table 3$)$. Race-stratified analyses suggested that this effect was more pronounced in EAs (ORT3 vs. T1 1.96; 95\% CI 1.23-3.12; $p$-trend $=0.003$ ) than AAs (ORT3 vs. T1 1.25; 95\% CI 0.81-1.93; p-trend=0.321), but there was no significant interaction by race ( $p$-interaction $=0.452)$. We observed an inverse association between high total fat-adjusted PUFA intake and PC aggressiveness among all research subjects $\left(\mathrm{OR}_{\mathrm{T} 3}\right.$ vs. T1 0.75 ; 95\% CI 0.55-1.03; $p$-trend $=0.075$ ), with a similar magnitude of inverse association in both EAs and AAs although neither overall nor race-stratified findings were statistically significant (Table 3). There were no significant associations between total fat-adjusted MUFA or trans fat intake and PC aggressiveness, overall or in race-stratified analyses. The magnitude and direction of association between tertiles of energy-adjusted total fat intake and PC aggressiveness was similar to that reported for total fat-adjusted saturated fat intake (Table 3). The use of race-specific tertiles of dietary fat intake did not substantially impact our findings (Supplementary Table 2).

\section{Total fat-adjusted cholesterol intake and PC aggressiveness}

High total fat-adjusted cholesterol intake was associated with increased PC aggressiveness in EAs (ORT3 vs. T1 1.62; 95\% CI 1.02-2.58; $p$-trend $=0.056)$ but not $\mathrm{AAs}(\mathrm{OR}$ T3 vs. T1 $0.92 ; 95 \% \mathrm{CI}$ $0.60-1.42 ; p$-trend $=0.750)$, although the interaction by race was not statistically significant $(p$ interaction $=0.244$ ). Analyses using race-specific tertiles of total fat-adjusted cholesterol intake produced similar findings (Supplementary Table 2). 
Statin use as an effect modifier of the saturated fat - PC aggressiveness association

Analyses stratified by statin use showed that research subjects in the upper tertile of total fatadjusted saturated fat intake had an elevated OR for high aggressive PC regardless of statin use status. However, there was a weaker association in statin users $\left(\mathrm{OR}_{\mathrm{T} 3}\right.$ vs. T1 1.16 ; $95 \%$ CI 0.67 $2.01 ; p$-trend $=0.661)$ compared to non-users (ORT3 vs. T1 $1.71 ; 95 \%$ CI 1.16-2.51; $p$-trend $=0.053$; Table 4), although there was no significant interaction by statin use ( $p$-interaction $=0.430)$. 


\section{Discussion}

Using data from the population-based, case-only PCaP, we report that elevated dietary intake of saturated fat was associated with increased PC aggressiveness. In secondary analysis, we found that higher total fat intake was also associated with more aggressive disease. Although there was no strong evidence for racial differences in these associations, effect estimates for total and saturated fat were larger in EAs relative to AAs, and high dietary cholesterol was associated with higher PC aggressiveness only in EAs. These findings support a role for dietary fat intake in PC aggressiveness in both EA and AA men, although future studies are required to test the suggestion that these associations may be stronger in EAs.

Relatively few studies to date have examined associations between dietary fat and PC by race. The prospective Multiethnic Cohort (MEC) study reported no associations between any type of dietary fat or cholesterol intake and either total or aggressive PC risk in any of five racial/ethnic groups examined ${ }^{5}$. A case-control study of four different racial/ethnic groups in North America reported a positive association between saturated fat intake and PC risk in both EAs and $\mathrm{AAs}^{20}$. Two studies indirectly assessed saturated fat intake by measuring red meat and animal fat consumption; one multi-center case-control study in the US showed a positive association between animal fat intake and aggressive disease in both EAs and $\mathrm{AAs}^{21}$, while the prospective Cancer Prevention Study II reported stronger associations between red meat consumption and PC risk in $\mathrm{AAs}^{22}$. Collectively, the findings of these prior studies and those of the present analysis do not provide strong evidence for racial differences in associations between dietary fat and cholesterol intake and PC aggressiveness and if a racial difference exists, the difference appears small. 
A number of biologic mechanisms may contribute to associations between dietary fat and PC aggressiveness. High fat diets can modulate androgen signaling ${ }^{23}$, up-regulate the insulin-like growth factor (IGF) pathway, and increase prostate inflammation, thereby promoting PC growth ${ }^{24,25}$. Moreover, saturated fat intake is an important dietary determinant of serum cholesterol levels ${ }^{12,26}$. Epidemiologic data support a positive association between high serum cholesterol and PC aggressiveness ${ }^{27}$ and an inverse association between statin use and PC aggressiveness ${ }^{15,28}$. If saturated fat intake promotes PC aggressiveness by raising serum cholesterol levels, the association between saturated fat and PC aggressiveness should be attenuated in individuals using statins to control serum cholesterol levels. We observed a weaker magnitude of effect in statin users, but found no strong evidence to support statin use as an effect modifier of the saturated fat-PC aggressiveness association. These findings may reflect the complex relationship between saturated fat and PC aggressiveness, given multiple determinants (both dietary and genetic) of serum cholesterol levels, in addition to the aforementioned cholesterol-independent mechanisms by which saturated fat may impact PC aggressiveness.

Epidemiologic evidence supporting an association between PUFA intake and PC aggressiveness is sparse and inconsistent. Individual studies have reported positive ${ }^{6}$ inverse $^{29}$ and null associations $^{8}$, and a recent meta-analysis reported a null association between PUFA intake and risk of both total and aggressive $\mathrm{PC}^{7}$. Furthermore, a recent systematic review found no consistent role for fish oil, an important source of omega-3 PUFAs, in reducing PC incidence, aggressiveness, or mortality ${ }^{30}$. In the present study, although the direction of association between PUFA intake and PC aggressiveness was inverse, these findings were not statistically significant either overall or in race-stratified analysis. 
Several limitations of this study should be considered. First, although we used a validated FFQ in the present study, FFQs are subject to recall bias and may be influenced by case status of the research subject. However, given that all of our research subjects were incident PC cases, this potential source of bias is likely to be minimal. Furthermore, the use of rapid case ascertainment limited the time between diagnosis and enrollment in PCaP, thus reducing the likelihood of bias from time or treatment. Second, serum levels of fatty acids and cholesterol were not measured in $\mathrm{PCaP}$ as measurements at the time of diagnosis may be impacted by presence of $\mathrm{PC}$, raising concerns about reverse causality ${ }^{31}$. Measurement of dietary fat intake using a validated FFQ may be less subject to this potential source of bias. These limitations are balanced by several important strengths. All types of dietary fat and cholesterol were adjusted for total fat intake using the residual method, and models were adjusted for energy-adjusted total fat intake and energy intake. This approach separated the effects of each fat type from total fat on PC aggressiveness while controlling for energy intake. Finally, an important strength of this study is the inclusion of both EAs and AAs in similar proportions, in addition to our comprehensive assessment of clinical and demographic characteristics and adjustment for these potential confounders in our analysis.

In summary, elevated dietary intake of saturated and total fat were associated with increased PC aggressiveness. Although we observed no statistically significant racial differences in these associations, high saturated and total fat intake were suggestively more strongly associated with PC aggressiveness in EAs, and cholesterol was associated with higher PC aggressiveness in EAs but not in AAs. Established PC risk factors, including older age, AA race, and family history of PC, are not modifiable. If confirmed, these findings may be relevant for aggressive PC prevention efforts, given that dietary fat content and composition are modifiable risk factors. 
Acknowledgements: The authors thank the staff, advisory committees and research subjects participating in the $\mathrm{PCaP}$ study for their important contributions.

Conflict of interest: The authors declare no conflict of interest 


\section{References}

1. Center MM, Jemal A, Lortet-Tieulent J, Ward E, Ferlay J, Brawley O et al. International variation in prostate cancer incidence and mortality rates. European urology 2012; 61(6): 1079-1092.

2. Micha R, Khatibzadeh S, Shi P, Fahimi S, Lim S, Andrews KG et al. Global, regional, and national consumption levels of dietary fats and oils in 1990 and 2010: a systematic analysis including 266 country-specific nutrition surveys. BMJ 2014; 348: g2272.

3. Kobayashi N, Barnard RJ, Said J, Hong-Gonzalez J, Corman DM, Ku M et al. Effect of low-fat diet on development of prostate cancer and Akt phosphorylation in the Hi-Myc transgenic mouse model. Cancer Res 2008; 68(8): 3066-3073.

4. Allott EH, Hursting SD. Obesity and cancer: mechanistic insights from transdisciplinary studies. Endocrine-related cancer 2015; 22(6): R365-386.

5. Park SY, Murphy SP, Wilkens LR, Henderson BE, Kolonel LN. Fat and meat intake and prostate cancer risk: the multiethnic cohort study. Int J Cancer 2007; 121(6): 1339-1345.

6. Kristal AR, Arnold KB, Neuhouser ML, Goodman P, Platz EA, Albanes D et al. Diet, supplement use, and prostate cancer risk: results from the prostate cancer prevention trial. American journal of epidemiology 2010; 172(5): 566-577.

7. Xu C, Han FF, Zeng XT, Liu TZ, Li S, Gao ZY. Fat Intake Is Not Linked to Prostate Cancer: A Systematic Review and Dose-Response Meta-Analysis. PloS one 2015; 10(7): e0131747.

8. Pelser C, Mondul AM, Hollenbeck AR, Park Y. Dietary fat, fatty acids, and risk of prostate cancer in the NIH-AARP diet and health study. Cancer Epidemiol Biomarkers Prev 2013; 22(4): 697-707.

9. Giovannucci E, Rimm EB, Colditz GA, Stampfer MJ, Ascherio A, Chute CG et al. A prospective study of dietary fat and risk of prostate cancer. Journal of the National Cancer Institute 1993; 85(19): 1571-1579.

10. Epstein MM, Kasperzyk JL, Mucci LA, Giovannucci E, Price A, Wolk A et al. Dietary fatty acid intake and prostate cancer survival in Orebro County, Sweden. American journal of epidemiology 2012; 176(3): 240-252.

11. Van Blarigan EL, Kenfield SA, Yang M, Sesso HD, Ma J, Stampfer MJ et al. Fat intake after prostate cancer diagnosis and mortality in the Physicians' Health Study. Cancer causes \& control : CCC 2015; 26(8): 1117-1126. 
12. Grundy SM, Vega GL. Causes of high blood cholesterol. Circulation 1990; 81(2): 412-427.

13. Allott EH, Howard LE, Cooperberg MR, Kane CJ, Aronson WJ, Terris MK et al. Postoperative statin use and risk of biochemical recurrence following radical prostatectomy: results from the Shared Equal Access Regional Cancer Hospital (SEARCH) database. BJU international 2014.

14. Allott EH, Howard LE, Cooperberg MR, Kane CJ, Aronson WJ, Terris MK et al. Serum Lipid Profile and Risk of Prostate Cancer Recurrence: Results from the SEARCH Database. Cancer Epidemiol Biomarkers Prev 2014; 23(11): 2349-2356.

15. Allott EH, Farnan L, Steck SE, Arab L, J.L. S, Mishel M et al. Statin use and prostate cancer aggressiveness: results from the population-based North Carolina-Louisiana Prostate Cancer Project. Cancer Epidemiol Biomarkers Prev 2016.

16. Schroeder JC, Bensen JT, Su L, Mishel M, Ivanova A, Smith GJ et al. The North Carolina-Louisiana Prostate Cancer Project (PCaP): methods and design of a multidisciplinary population-based cohort study of racial differences in prostate cancer outcomes. The Prostate 2006; 66(11): 11621176.

17. Subar AF, Thompson FE, Kipnis V, Midthune D, Hurwitz P, McNutt S et al. Comparative validation of the Block, Willett, and National Cancer Institute food frequency questionnaires : the Eating at America's Table Study. American journal of epidemiology 2001; 154(12): 1089-1099.

18. Thompson FE, Subar AF, Brown CC, Smith AF, Sharbaugh CO, Jobe JB et al. Cognitive research enhances accuracy of food frequency questionnaire reports: results of an experimental validation study. Journal of the American Dietetic Association 2002; 102(2): 212-225.

19. Willett W, Stampfer MJ. Total energy intake: implications for epidemiologic analyses. American journal of epidemiology 1986; 124(1): 17-27.

20. Whittemore AS, Kolonel LN, Wu AH, John EM, Gallagher RP, Howe GR et al. Prostate cancer in relation to diet, physical activity, and body size in blacks, whites, and Asians in the United States and Canada. Journal of the National Cancer Institute 1995; 87(9): 652-661.

21. Hayes RB, Ziegler RG, Gridley G, Swanson C, Greenberg RS, Swanson GM et al. Dietary factors and risks for prostate cancer among blacks and whites in the United States. Cancer Epidemiol Biomarkers Prev 1999; 8(1): 25-34. 
22. Rodriguez C, McCullough ML, Mondul AM, Jacobs EJ, Chao A, Patel AV et al. Meat consumption among Black and White men and risk of prostate cancer in the Cancer Prevention Study II Nutrition Cohort. Cancer Epidemiol Biomarkers Prev 2006; 15(2): 211-216.

23. Gromadzka-Ostrowska J. Effects of dietary fat on androgen secretion and metabolism. Reprod Biol 2006; 6 Suppl 2: 13-20.

24. Shankar E, Bhaskaran N, MacLennan GT, Liu G, Daneshgari F, Gupta S. Inflammatory Signaling Involved in High-Fat Diet Induced Prostate Diseases. J Urol Res 2015; 2(1).

25. Di Sebastiano KM, Mourtzakis M. The role of dietary fat throughout the prostate cancer trajectory. Nutrients 2014; 6(12): 6095-6109.

26. Mensink RP, Zock PL, Kester AD, Katan MB. Effects of dietary fatty acids and carbohydrates on the ratio of serum total to HDL cholesterol and on serum lipids and apolipoproteins: a metaanalysis of 60 controlled trials. Am J Clin Nutr 2003; 77(5): 1146-1155.

27. Platz EA, Till C, Goodman PJ, Parnes HL, Figg WD, Albanes D et al. Men with low serum cholesterol have a lower risk of high-grade prostate cancer in the placebo arm of the prostate cancer prevention trial. Cancer Epidemiol Biomarkers Prev 2009; 18(11): 2807-2813.

28. Platz EA, Leitzmann MF, Visvanathan K, Rimm EB, Stampfer MJ, Willett WC et al. Statin drugs and risk of advanced prostate cancer. Journal of the National Cancer Institute 2006; 98(24): 1819-1825.

29. Agalliu I, Kirsh VA, Kreiger N, Soskolne CL, Rohan TE. Oxidative balance score and risk of prostate cancer: results from a case-cohort study. Cancer epidemiology 2011; 35(4): 353-361.

30. Lovegrove C, Ahmed K, Challacombe B, Khan MS, Popert R, Dasgupta P. Systematic review of prostate cancer risk and association with consumption of fish and fish-oils: analysis of 495,321 participants. International journal of clinical practice 2015; 69(1): 87-105.

31. Ahn J, Lim U, Weinstein SJ, Schatzkin A, Hayes RB, Virtamo J et al. Prediagnostic total and highdensity lipoprotein cholesterol and risk of cancer. Cancer Epidemiol Biomarkers Prev 2009; 18(11): 2814-2821. 
Table 1: Characteristics of research subjects with low/intermediate versus high aggressive prostate cancer

\begin{tabular}{|c|c|c|c|}
\hline & \multicolumn{2}{|c|}{ Prostate cancer aggressiveness } & \multirow[b]{2}{*}{ p-value } \\
\hline & Low/intermediate & High & \\
\hline Age at diagnosis, mean (SD) & $62.6(7.9)$ & $64.7(7.6)$ & $<0.0001$ \\
\hline Race, n (\%) & & & \multirow{3}{*}{0.001} \\
\hline European American & $847(55)$ & $146(45)$ & \\
\hline African American & $686(45)$ & $175(55)$ & \\
\hline Site, n (\%) & & & \multirow[b]{3}{*}{0.050} \\
\hline North Carolina & $751(49)$ & $138(43)$ & \\
\hline Louisiana & $782(51)$ & $183(57)$ & \\
\hline Marital status, n (\%) & & & \multirow{4}{*}{$<0.0001$} \\
\hline Single/never married & $58(4)$ & $21(7)$ & \\
\hline Divorced/separated/widowed & $260(17)$ & $82(26)$ & \\
\hline Married/living as married & $1,215(79)$ & $218(68)$ & \\
\hline Screening frequency, n (\%) & & & \multirow{4}{*}{$<0.0001$} \\
\hline Never & $234(15)$ & $98(31)$ & \\
\hline$\leq 1$ PSA/DRE test per year & $947(62)$ & $167(52)$ & \\
\hline$>1$ PSA/DRE test per year & $352(23)$ & $56(17)$ & \\
\hline Education, n (\%) & & & \multirow{4}{*}{$<0.0001$} \\
\hline Less than high school & $249(16)$ & $88(27)$ & \\
\hline High school graduate & $378(25)$ & $69(22)$ & \\
\hline College graduate or some college & $905(59)$ & $164(51)$ & \\
\hline Income $^{\ddagger},(\%)$ & & & \multirow{5}{*}{$<0.0001$} \\
\hline$<\$ 20,000$ & $246(17)$ & $89(31)$ & \\
\hline$\$ 20,000-\$ 50,000$ & $476(34)$ & $95(33)$ & \\
\hline$\$ 50,000-\$ 80,000$ & $309(22)$ & $47(16)$ & \\
\hline$>\$ 80,000$ & $378(27)$ & $57(20)$ & \\
\hline Smoking status, n (\%) & & & \multirow{4}{*}{$<0.0001$} \\
\hline Never & $537(35)$ & $89(28)$ & \\
\hline Past & $796(52)$ & $165(51)$ & \\
\hline Current & $200(13)$ & $67(21)$ & \\
\hline BMI $\left(\mathrm{kg} / \mathrm{m}^{2}\right), \mathrm{n}(\%)$ & & & \multirow{4}{*}{0.041} \\
\hline$<25$ & 287 (19) & $55(17)$ & \\
\hline $25-30$ & $678(44)$ & $123(38)$ & \\
\hline$\geq 30$ & $568(37)$ & $143(45)$ & \\
\hline Statin use, $n(\%)$ & & & \multirow{3}{*}{0.049} \\
\hline No & $932(61)$ & $214(67)$ & \\
\hline Yes & $601(39)$ & $107(33)$ & \\
\hline Saturated fat (\% total fat), mean (SD) & $31.0(4.5)$ & $31.9(4.7)$ & 0.001 \\
\hline PUFA (\% total fat), mean (SD) & $23.3(4.2)$ & $22.5(4.4)$ & 0.005 \\
\hline MUFA (\% total fat), mean (SD) & $38.2(2.6)$ & $38.1(2.4)$ & 0.747 \\
\hline Trans fat (\% total fat), mean (SD) & $6.2(1.7)$ & $6.3(1.7)$ & 0.409 \\
\hline Total fat (\% energy), mean (SD) & $34.0(6.8)$ & $34.9(7.1)$ & 0.040 \\
\hline Cholesterol (mg/day), median (IQR) & $272(186-371)$ & $304(222-421)$ & 0.0001 \\
\hline Energy intake (kcal/day), median (IQR) & $2,253(1,713-3,000)$ & $2,450(1,802-3,324)$ & 0.006 \\
\hline
\end{tabular}


${ }^{\ddagger} \mathrm{n}=41$ did not know their income and $\mathrm{n}=116$ refused to answer this question; $\mathrm{n}=1$ was missing education 
Table 2: Characteristics of research subjects by tertile of total fat-adjusted saturated fat intake

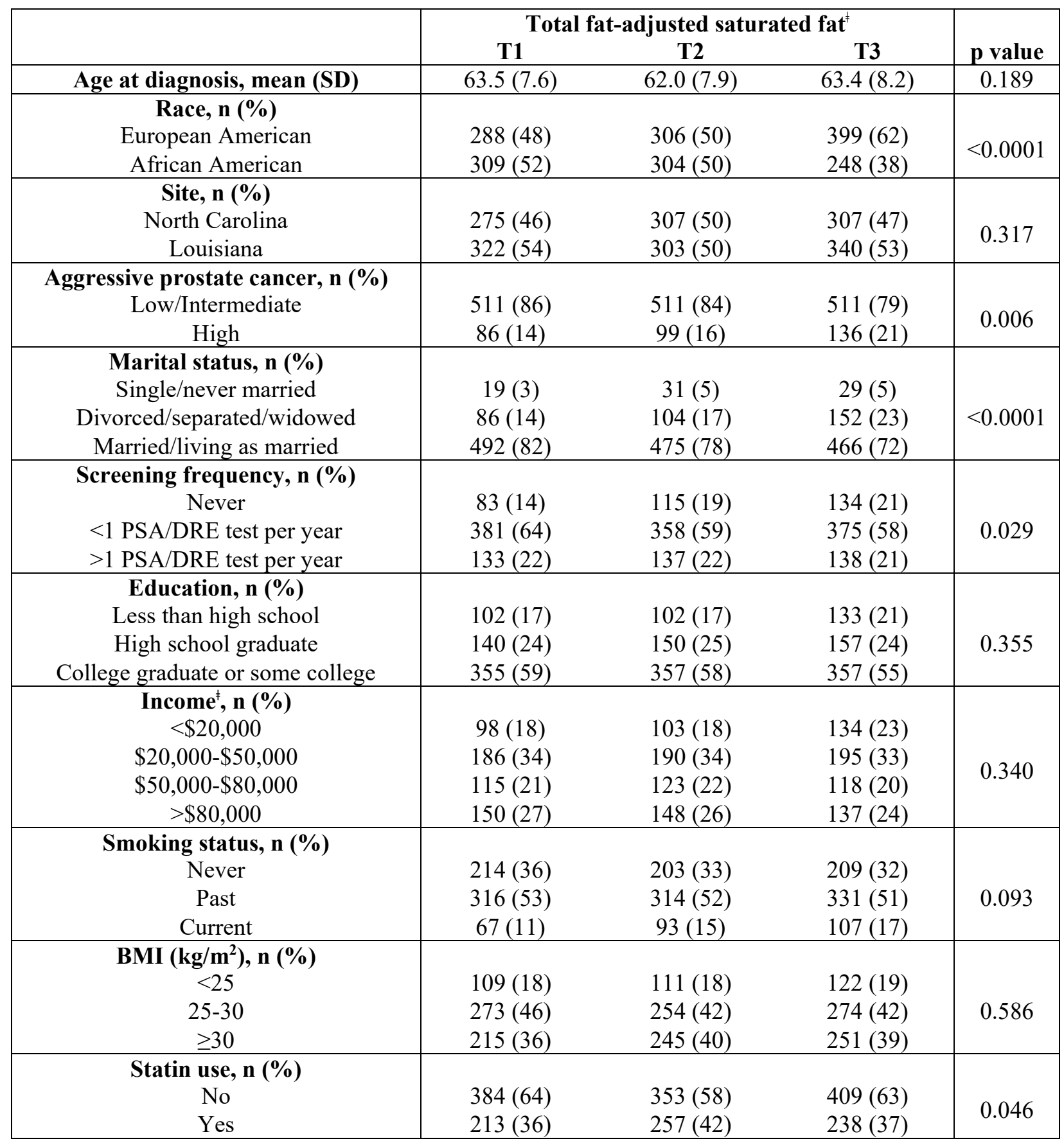

${ }^{\ddagger} \mathrm{n}=41$ did not know their income and $\mathrm{n}=116$ refused to answer this question; $\mathrm{n}=1$ was missing education *saturated fat was adjusted for total fat intake using the residual method 
Table 3: Odds ratios for aggressive prostate cancer according to tertiles of total fat-adjusted dietary fat and cholesterol intake, overall and stratified by race

\begin{tabular}{|c|c|c|c|c|c|c|}
\hline & \multicolumn{2}{|c|}{ All } & \multicolumn{2}{|c|}{ European American } & \multicolumn{2}{|c|}{ African American } \\
\hline & $\begin{array}{c}n, \\
\text { low/intermediate } \\
\text { (high aggressive) }\end{array}$ & $\begin{array}{c}\text { OR }^{*} \\
(95 \% \text { CI })\end{array}$ & $\begin{array}{c}\text { n, } \\
\text { low/intermediate } \\
\text { (high aggressive) }\end{array}$ & $\begin{array}{c}\text { OR }^{*} \\
(95 \% \text { CI })\end{array}$ & $\begin{array}{c}\text { n, } \\
\text { low/intermediate } \\
\text { (high aggressive) }\end{array}$ & $\begin{array}{c}\text { OR* } \\
(95 \% \text { CI })\end{array}$ \\
\hline \multicolumn{7}{|l|}{ Saturated fat ${ }^{\ddagger}$} \\
\hline $\mathrm{T} 1$ & $511(86)$ & 1.00 (ref) & $256(32)$ & 1.00 (ref) & $255(54)$ & 1.00 (ref) \\
\hline $\mathrm{T} 2$ & $511(99)$ & $1.17(0.84-1.62)$ & $268(38)$ & $1.23(0.73-2.07)$ & $243(61)$ & $1.05(0.68-1.63)$ \\
\hline $\mathrm{T} 3$ & $511(136)$ & $1.51(1.10-2.06)$ & $323(76)$ & $1.96(1.23-3.12)$ & $188(60)$ & $1.25(0.81-1.93)$ \\
\hline p-trend & & 0.009 & & 0.003 & & 0.321 \\
\hline \multicolumn{7}{|l|}{$P U F A^{\ddagger}$} \\
\hline $\mathrm{T} 1$ & $511(126)$ & 1.00 (ref) & $314(61)$ & 1.00 (ref) & $197(65)$ & 1.00 (ref) \\
\hline $\mathrm{T} 2$ & $511(106)$ & $0.89(0.66-1.20)$ & $262(47)$ & $0.99(0.64-1.53)$ & $249(59)$ & $0.78(0.51-1.19)$ \\
\hline $\mathrm{T} 3$ & $511(89)$ & $0.75(0.55-1.03)$ & $271(38)$ & $0.70(0.44-1.11)$ & $240(51)$ & $0.73(0.48-1.13)$ \\
\hline p-trend & & 0.075 & & 0.154 & & 0.157 \\
\hline \multicolumn{7}{|l|}{ MUFA $A^{\ddagger}$} \\
\hline $\mathrm{T} 1$ & $511(107)$ & 1.00 (ref) & $297(53)$ & 1.00 (ref) & $214(54)$ & 1.00 (ref) \\
\hline $\mathrm{T} 2$ & $511(118)$ & $1.03(0.77-1.40)$ & $282(56)$ & $1.07(0.70-1.65)$ & $229(62)$ & $0.95(0.61-1.46)$ \\
\hline $\mathrm{T} 3$ & $511(96)$ & $0.89(0.65-1.22)$ & $268(37)$ & $0.81(0.50-1.30)$ & $243(59)$ & $0.99(0.64-1.53)$ \\
\hline p-trend & & 0.509 & & 0.436 & & 0.956 \\
\hline \multicolumn{7}{|l|}{ Trans fat } \\
\hline $\mathrm{T} 1$ & $511(101)$ & 1.00 (ref) & $332(58)$ & 1.00 (ref) & $179(43)$ & $1.00($ ref) \\
\hline $\mathrm{T} 2$ & $511(109)$ & $0.98(0.72-1.33)$ & $270(55)$ & $1.20(0.79-1.83)$ & $241(54)$ & $0.82(0.51-1.30)$ \\
\hline $\mathrm{T} 3$ & $511(111)$ & $0.97(0.71-1.33)$ & $245(33)$ & $0.76(0.47-1.23)$ & $266(78)$ & $1.09(0.70-1.70)$ \\
\hline p-trend & & 0.857 & & 0.376 & & 0.584 \\
\hline \multicolumn{7}{|l|}{ Total fat ${ }^{\ddagger}$} \\
\hline $\mathrm{T} 1$ & $511(96)$ & 1.00 (ref) & $230(28)$ & 1.00 (ref) & $281(68)$ & 1.00 (ref) \\
\hline $\mathrm{T} 2$ & $511(99)$ & $1.12(0.82-1.55)$ & $287(42)$ & $1.18(0.70-1.99)$ & $224(57)$ & $1.14(0.75-1.72)$ \\
\hline $\mathrm{T} 3$ & $511(126)$ & $1.48(1.08-2.02)$ & $330(76)$ & $1.84(1.13-2.98)$ & $181(50)$ & $1.19(0.77-1.83)$ \\
\hline p-trend & & 0.016 & & 0.010 & & 0.408 \\
\hline \multicolumn{7}{|l|}{ Cholesterol $^{\ddagger}$} \\
\hline $\begin{array}{l}11 \\
\mathrm{~T} 2\end{array}$ & $\begin{array}{l}511(94) \\
511(98)\end{array}$ & $\begin{array}{c}1.00(\text { ref) } \\
0.92(0.66-1.26)\end{array}$ & $\begin{array}{l}319(49) \\
311(47)\end{array}$ & $\begin{array}{c}1.00(\mathrm{ref}) \\
1.02(0.65-1.59)\end{array}$ & $\begin{array}{l}192(45) \\
200(51)\end{array}$ & $\begin{array}{c}1.00(\text { ref }) \\
0.85(0.53-1.36)\end{array}$ \\
\hline
\end{tabular}




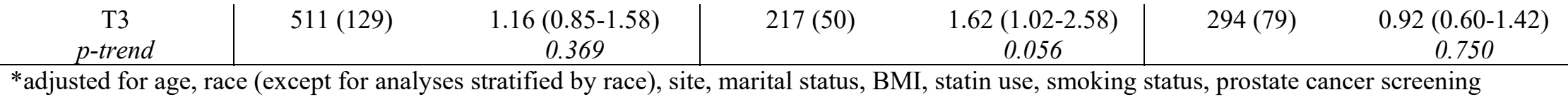
frequency, energy-adjusted total fat intake (except for total fat analyses) and energy intake

fadjusted for total fat intake using the residual method (with the exception of total fat which was adjusted for energy intake using the residual method) 
Table 4: Associations between tertiles of total fat-adjusted saturated fat intake and prostate cancer aggressiveness stratified by statin use

\begin{tabular}{|c|c|c|c|c|}
\hline & \multicolumn{2}{|c|}{ Statin non-users } & \multicolumn{2}{|c|}{ Statin users } \\
\hline & $\begin{array}{l}\mathrm{n}, \\
\text { low/intermediate } \\
\text { (high aggressive) }\end{array}$ & $O R *(95 \% C I)$ & $\begin{array}{c}n, \\
\text { low/intermediate } \\
\text { (high aggressive) }\end{array}$ & $O R *(95 \% C I)$ \\
\hline Saturated fat ${ }^{\ddagger}$ & & & & \\
\hline $\mathrm{T} 1$ & $328(56)$ & 1.00 (ref) & $183(30)$ & $1.00(\mathrm{ref})$ \\
\hline $\mathrm{T} 2$ & $292(61)$ & $1.21(0.80-1.84)$ & $219(38)$ & $1.03(0.60-1.78)$ \\
\hline $\mathrm{T} 3$ & 312 (97) & $1.71(1.16-2.51)$ & 199 (39) & $1.16(0.67-2.01)$ \\
\hline$p$-trend & & 0.053 & & 0.661 \\
\hline
\end{tabular}

*adjusted for age, race, site, marital status, BMI, smoking status, prostate cancer screening frequency, energy-adjusted total fat intake, and energy intake

"saturated fat was adjusted for total fat intake using the residual method 